\title{
Vector-valued wavelets and the Hardy space $H^{1}\left(\mathbb{R}^{n}, X\right)$
}

\author{
by \\ TuOmas Hytönen (Turku)
}

\begin{abstract}
We prove an analogue of Y. Meyer's wavelet characterization of the Hardy space $H^{1}\left(\mathbb{R}^{n}\right)$ for the space $H^{1}\left(\mathbb{R}^{n}, X\right)$ of $X$-valued functions. Here $X$ is a Banach space with the UMD property. The proof uses results of T. Figiel on generalized CalderónZygmund operators on Bochner spaces and some new local estimates.
\end{abstract}

1. Introduction. One of the main aspects of the theory of Hardy spaces is the equivalence of their various definitions. In the real-variable theory, one finds (at least) four major types of characterizations of $H^{1}$ : integrability of maximal functions, integrability of square functions, integrability of conjugate functions (the Hilbert transform, or its variants), and atomic decompositions. In the present paper, we contribute to this theory by establishing a number of characterizations of the square function type for the Hardy space $H^{1}\left(\mathbb{R}^{n}, X\right)$ of Banach-space-valued functions, which is initially defined in terms of the atomic decomposition as follows:

We say that $f \in H^{1}\left(\mathbb{R}^{n}, X\right)$ if $f \in L^{1}\left(\mathbb{R}^{n}, X\right)$ has an expansion

$$
f(x)=\sum_{i=1}^{\infty} a_{i}(x), \quad \operatorname{supp} a_{i} \subset \bar{B}_{i}, \quad \int a_{i}(x) d x=0,
$$

where the $\bar{B}_{i}$ are balls in $\mathbb{R}^{n}$, and we have

$$
\sum_{i=1}^{\infty}\left\|a_{i}\right\|_{L^{p}\left(\mathbb{R}^{n}, X\right)}\left|\bar{B}_{i}\right|^{1 / p^{\prime}}<\infty
$$

where $p \in] 1, \infty\left[\right.$ is fixed, and $p^{\prime}$ denotes the conjugate exponent, $1 / p+1 / p^{\prime}=1$. The norm $\|f\|_{H^{1}\left(\mathbb{R}^{n}, X\right)}$ is defined as the infimum of the values (1.1) taken over all such decompositions. Its numerical value depends on the choice of $p \in] 1, \infty[$, but it is well known that each $p \in] 1, \infty[$ (actually also $p=\infty$ ) gives the same space $H^{1}\left(\mathbb{R}^{n}, X\right)$ with an equivalent norm (cf. [16] for a treatment in the vector-valued setting).

2000 Mathematics Subject Classification: 42B30, 42C40, 46E40.

Key words and phrases: wavelet basis, atomic decomposition, generalized CalderónZygmund operators, UMD space. 
Let us note that this atomic definition of $H^{1}\left(\mathbb{R}^{n}, X\right)$ is known to agree with one given in terms of various maximal functions, even for an arbitrary Banach space $X$. Actually one can check that the proof of this fact in the scalar case, as given e.g. in E. M. Stein's book [21], goes through word for word in the general setting. For $n=1$, the "conjugate Hardy space", defined as the domain of the Hilbert transform on $L^{1}(\mathbb{R}, X)$ with the graph norm, is always (i.e., without any conditions on the Banach space $X$ ) contained in the atomic Hardy space, and agrees with it exactly when $X$ is a UMD space. (See the papers of O. Blasco [1], J. Bourgain [3] and D. L. Burkholder [5] in this connection.) None of these results, however, will play a rôle in the proof of our main characterization theorem, but we always work with the atomic definition.

The square function description of $H^{1}\left(\mathbb{R}^{n}, X\right)$ that we have in mind involves the wavelet expansion of a function, and extends Y. Meyer's [18] characterization of $H^{1}\left(\mathbb{R}^{n}\right)$. Recall (cf. [18] for more details) that a wavelet basis of $L^{2}\left(\mathbb{R}^{n}\right)$ is a complete orthonormal system $\left(\psi_{\lambda}\right)_{\lambda \in \Lambda}$, where $\Lambda$ is the set of dyadic $n$-vectors of the form $\lambda=k 2^{-j}+\eta 2^{-j-1}\left(j \in \mathbb{Z}, k \in \mathbb{Z}^{n}\right.$, $\left.\eta \in\{0,1\}^{n} \backslash\{0\}\right)$, and $\psi_{\lambda}(x)=2^{j n / 2} \psi^{\eta}\left(2^{j} x-k\right)$, where $\psi^{\eta} \in L^{2}\left(\mathbb{R}^{n}\right)$, $\eta \in\{0,1\}^{n} \backslash\{0\}$, are the $2^{n}-1$ mother wavelets. The basis is called $r$-regular if $\left|\partial^{\alpha} \psi^{\eta}(x)\right| \leq C_{m}(1+|x|)^{-m}$ and $\int x^{\alpha} \psi^{\eta}(x) d x=0$ for all $|\alpha| \leq r$, all $m \in \mathbb{N}$ and all $\eta \in\{0,1\}^{n} \backslash\{0\}$. Meyer's theorem is the following:

1.2. THEOREM ([18]). Let $\left(\psi_{\lambda}\right)_{\lambda \in \Lambda}$ be a 1 -regular wavelet basis of $L^{2}\left(\mathbb{R}^{n}\right)$. The following conditions are equivalent for the distribution $f(x)=$ $\sum_{\lambda \in \Lambda} \alpha_{\lambda} \psi_{\lambda}(x)$ :

$$
\begin{aligned}
& f \in H^{1}\left(\mathbb{R}^{n}\right), \\
& \sup _{F \subset \Lambda} \sup _{\varepsilon \in\{ \pm 1\}^{\Lambda}}\left\|\sum_{\lambda \in F} \varepsilon_{\lambda} \alpha_{\lambda} \psi_{\lambda}(\cdot)\right\|_{L^{1}\left(\mathbb{R}^{n}\right)}<\infty \\
& \left(\sum_{\lambda \in \Lambda}\left|\alpha_{\lambda}\right|^{2}\left|\psi_{\lambda}(\cdot)\right|^{2}\right)^{1 / 2} \in L^{1}\left(\mathbb{R}^{n}\right), \\
& \left(\sum_{\lambda \in \Lambda}\left|\alpha_{\lambda}\right|^{2}|Q(\lambda)|^{-1} 1_{Q(\lambda)}(\cdot)\right)^{1 / 2} \in L^{1}\left(\mathbb{R}^{n}\right), \\
& \left(\sum_{\lambda \in \Lambda}|\alpha(\lambda)|^{2}|Q(\lambda)|^{-1} 1_{R(\lambda)}(\cdot)\right)^{1 / 2} \in L^{1}\left(\mathbb{R}^{n}\right),
\end{aligned}
$$

where

- the first supremum in (1.4) is taken over all finite subsets $F$ of $\Lambda$,

- $Q(\lambda):=2^{-j}\left(\left[0,1\left[^{n}+k\right)\right.\right.$ for $\lambda=k 2^{-j}+\eta 2^{-j-1}$,

- $R(\lambda):=2^{-j}\left(A^{\eta}+k\right)$, where $A^{\eta}$ is any non-degenerate cube. 
For historical reflection, we note that Meyer's theorem was preceded by a similar characterization using spline bases of order $r \geq 2$ obtained by S.-Y. A. Chang and Z. Ciesielski [7]. Moreover, the direction from the square function estimates to the atomic decomposition in Meyer's proof is a variant of ideas that already appeared in similar situations in a number of earlier works, apparently for the first time in A. P. Calderón's treatment of "parabolic" $H^{p}$ spaces [6], and then in the papers of Chang and R. Fefferman [8, 9].

Theorem 1.2 can also be viewed as a wavelet analogue of B. Davis' inequality for martingales [11], as both assert that the $L^{1}$ norm of a certain square function gives an equivalent norm on $H^{1}$. In fact, if we could, for $n=1$, take our wavelet basis to be the Haar system on $L^{2}(\mathbb{R})$, then the function appearing in (1.5), as well as that in (1.6), would be the martingale square function of $f$ with respect to the dyadic filtration of the real line. However, the Haar system, although a wavelet basis, is not 1-regular, and actually the square function condition just described does not characterize the membership of $f$ in $H^{1}(\mathbb{R})$ but in the smaller dyadic Hardy space, which indeed coincides with the martingale Hardy space related to the dyadic filtration (cf. [18]). Thus, while the results are analogous, they do not cover each other.

It is the martingale Hardy space that seems to have been more intensively studied in the vector-valued context, which is rather natural since the fundamental UMD condition-which one typically needs to impose on the Banach space $X$ in order to have some deeper-lying analytic results - is itself stated in terms of martingales. Recall that a Banach space $X$ is UMD if for some (and then all, cf. [5]) $1<p<\infty$ there is a finite constant $C$ so that

$$
\left\|\sum_{k=1}^{n} \epsilon_{k} d_{k}\right\|_{L^{p}(\Omega, X)} \leq C\left\|\sum_{k=1}^{n} d_{k}\right\|_{L^{p}(\Omega, X)}
$$

for all $n \in \mathbb{Z}_{+}$, whenever $\left(\epsilon_{k}\right)_{k=1}^{n} \in\{-1,+1\}^{n}$ and $\left(d_{k}\right)_{k=1}^{n} \in L^{p}(\Omega, X)^{n}$ is a martingale difference sequence on an arbitrary probability space $(\Omega, \mathcal{A}, \mu)$ (i.e., there are sub- $\sigma$-algebras $\mathcal{A}_{0} \subset \mathcal{A}_{1} \subset \cdots \subset \mathcal{A}_{n} \subset \mathcal{A}$ such that for all $k=1, \ldots, n$, the function $d_{k}$ is $\mathcal{A}_{k}$-measurable and $\int_{A} d_{k} d \mu=0$ for all $A \in \mathcal{A}_{k-1}$ ). P. F. X. Müller and G. Schechtman [20] have extended Davis' inequality, with the square function rewritten in terms of a Rademacher average, to the UMD-space-valued $H^{1}$.

Other vector-valued results closely related to the present investigation are due to T. Figiel [13]. He established the unconditionality of wavelet decompositions in $L^{p}\left(\mathbb{R}^{n}, X\right)$ when $X$ is a UMD space and $1<p<\infty$. While the result itself does not involve martingales, its proof is based on martingale techniques, and in particular on the unconditionality of the Haar system on $L^{p}\left(\mathbb{R}^{n}, X\right)$. This is an easy consequence of the UMD inequality, as the Haar functions (with $X$-valued coefficients) on $[0,1]$, in their natural 
ordering, constitute a martingale difference sequence for which (1.8) applies; the resulting estimate is readily transferred to the Haar system on $\mathbb{R}^{n}$. Let us also recall a theorem of B. Maurey [17], which asserts that the validity of (1.8) for this particular choice already implies the full UMD condition.

A similar approach based on the defining inequality (1.8) of UMD spaces does not seem available in the case of our interest, because of the already mentioned reason that the Haar system does not span the full $H^{1}\left(\mathbb{R}^{n}, X\right)$ space but only its dyadic analogue. On the contrary, we will be concerned with the Calderón-Zygmund-theoretic properties of UMD spaces, which were revealed by the works of D. L. Burkholder [4], J. Bourgain [3] and T. Figiel [14] in the 80's: They established the $L^{p}\left(\mathbb{R}^{n}, X\right)$-boundedness, respectively, of the Hilbert transform, of all singular convolution operators with a standard kernel, and finally of generalized Calderón-Zygmund operators as in the T(1) theorem of G. David and J.-L. Journé [10]. Conversely, Bourgain also showed that the Hilbert transform boundedness again implies the UMD condition (1.8).

We now come to the statement of our main theorem. As in Müller and Schechtman's formulation of the UMD-valued Davis's inequality, and in many other results of analysis of vector-valued functions, we replace the square functions in (1.5) through (1.7) by Rademacher averages. We denote by $\varepsilon_{\lambda}$ independent random variables on some probability space $\Omega$ with distribution $P\left(\varepsilon_{\lambda}=+1\right)=P\left(\varepsilon_{\lambda}=-1\right)=1 / 2 . E_{\varepsilon}$ denotes the corresponding expectation. Then we have:

1.9. TheOREM. Let $X$ be a UMD space, let $\left(\psi_{\lambda}\right)_{\lambda \in \Lambda}$ be a 1-regular wavelet basis of $L^{2}\left(\mathbb{R}^{n}\right)$, and $\alpha \in X^{\Lambda}$. The following conditions are equivalent for the $X$-valued distribution $f(x)=\sum_{\lambda \in \Lambda} \alpha_{\lambda} \psi_{\lambda}(x)$ :

$$
\begin{aligned}
& f \in H^{1}\left(\mathbb{R}^{n}, X\right), \\
& \sup _{F \subset \Lambda} \sup _{\varepsilon \in\{ \pm 1\}^{\Lambda}} \|\left.\sum_{\lambda \in F} \varepsilon_{\lambda} \alpha_{\lambda} \psi_{\lambda}(\cdot)\right|_{L^{1}\left(\mathbb{R}^{n}, X\right)}<\infty, \\
& \int_{\mathbb{R}^{n}} E_{\varepsilon}\left|\sum_{\lambda \in \Lambda} \varepsilon_{\lambda} \alpha_{\lambda} \psi_{\lambda}(x)\right|_{X} d x<\infty, \\
& \left.\left.\int_{\mathbb{R}^{n}} E_{\varepsilon}\left|\sum_{\lambda \in \Lambda} \varepsilon_{\lambda} \alpha_{\lambda}\right| Q(\lambda)\right|^{-1 / 2} 1_{Q(\lambda)}(x)\right|_{X} d x<\infty, \\
& \left.\left.\int_{\mathbb{R}^{n}} E_{\varepsilon}\left|\sum_{\lambda \in \Lambda} \varepsilon_{\lambda} \alpha_{\lambda}\right| Q(\lambda)\right|^{-1 / 2} 1_{R(\lambda)}(x)\right|_{X} d x<\infty,
\end{aligned}
$$

where $F, \lambda, Q(\lambda)$ and $R(\lambda)=2^{-j}\left(A^{\eta}+k\right)$ have the same meaning as in Theorem 1.2. Moreover, the expressions (1.11) through (1.14) define equivalent norms on $H^{1}\left(\mathbb{R}^{n}, X\right)$. Consequently, the wavelet series of $f$ converges unconditionally to $f$ in the $H^{1}\left(\mathbb{R}^{n}, X\right)$ norm. 
Note that the condition (1.14) a priori depends on the choice of the cubes $A^{\eta}$ defining the $R(\lambda)$ 's. However, the proof will show that the validity of this condition for any one choice of the $A^{\eta}$ 's already implies it for all possible choices. Also recall that (1.10) has an implicit dependence on the exponent $p \in] 1, \infty\left[\right.$ appearing in the definition of $H^{1}\left(\mathbb{R}^{n}, X\right)$, but no such dependence is present in the other four conditions. Thus, as a by-product, we also obtain a new proof of the $p$-independence of the atomic definition of $H^{1}\left(\mathbb{R}^{n}, X\right)$ when $X$ is a UMD space.

To simplify the proof of Theorem 1.9 to be given in the following sections, note that it suffices to establish the equivalence of the different norms in the case of $\left(\alpha_{\lambda}\right)_{\lambda \in \Lambda}$ finitely non-zero. The general case then follows by standard methods, using the density in $H^{1}\left(\mathbb{R}^{n}, X\right)$ of such functions.

The paper is organized as follows: In Sec. 2 we show that (1.10) implies the other conditions; the main arguments here are based on Figiel's $T 1$ theorem [14]. The reverse direction, which consists of constructing an atomic decomposition for a function $f$ satisfying a randomized "square-function" estimate, is given in Sec. 3 and involves some new local estimates. We conclude with a discussion of the vector-valued BMO space and its duality with $H^{1}$ in Sec. 4.

1.15. REMARK. The validity of Theorem 1.9 on $H^{1}(\mathbb{R}, X)$ actually characterizes the UMD property of $X$. Indeed, let $X$ be any complex Banach space and let the conclusions of Theorem 1.9 be satisfied. Let $\left(\psi_{\lambda}\right)_{\lambda \in \Lambda}$ be the Littlewood-Paley wavelet basis of Meyer [18]. Since $\widehat{\psi}_{\lambda} \in \mathcal{D}(\mathbb{R})$ is supported away from the origin (where the multiplier of the Hilbert transform has a discontinuity), and since the Hilbert transform is an isometry on $L^{2}(\mathbb{R})$, it follows that $\left(H \psi_{\lambda}\right)_{\lambda \in \Lambda}$, too, is an infinitely regular wavelet basis of $L^{2}(\mathbb{R})$.

But then, according to our assumption, the $H^{1}(\mathbb{R}, X)$ norms of both $f(x)=\sum \alpha_{\lambda} \psi_{\lambda}(x)$ and $H f(x)=\sum \alpha_{\lambda} H \psi_{\lambda}(x)$ are comparable (with constants independent of $f$ ) to the quantity (1.13). In particular, $\|H f\|_{H^{1}(\mathbb{R}, X)} \leq$ $C\|f\|_{H^{1}(\mathbb{R}, X)}$, and so $H$ is bounded on $H^{1}(\mathbb{R}, X)$. This is equivalent to its $L^{p}(\mathbb{R}, X)$-boundedness (a result due to Blasco [1]), and thus to the UMD property of $X$. Since Blasco's proof is given in the slightly different periodic setting, let us briefly indicate the argument for the present case: For an operator with a standard kernel, the $H^{1}(\mathbb{R}, X)$-boundedness implies boundedness from $L_{0}^{\infty}(\mathbb{R}, X)$ [compactly supported $L^{\infty}$ functions with vanishing integral, equipped with the norm of $\left.L^{\infty}(\mathbb{R}, X)\right]$ to $\operatorname{BMO}(\mathbb{R}, X)$. See [16, p. 49] for an argument valid in the vector-valued setting. Then we just use interpolation; again cf. [16].

Acknowledgments. I wish to thank Dr. Hans-Olav Tylli, who brought the results of T. Figiel to my attention; Prof. Tadeusz Figiel himself, who kindly supplied me with further pieces of his work; and Prof. Oscar Blasco, 
who asked me a question which led me to investigate the validity of Remark 1.15. The anonymous referee is acknowledged for bringing to my attention several references which helped elaborate the historical perspectives given in the Introduction. I express my thanks to the Magnus Ehrnrooth Foundation for financial support.

2. Implications using Calderón-Zygmund operators. In proving Theorem 1.9, we will need to apply several transformations of the wavelet series. All these transformations will have the generic form of an integral operator

$$
T f(x)=\int_{\mathbb{R}^{n}} k(x, y) f(y) d y,
$$

where the kernel $k$ is actually bounded and integrable. What is important is to obtain appropriate uniform bounds for operator norms of different operators $T$ of this kind.

T. Figiel [14] has generalized the famous $T 1$ theorem of G. David and J.-L. Journé to the setting of $X$-valued $L^{p}$ spaces. (See also [15], where an intermediate estimate omitted in [14] is proved in detail.) A rather general formulation of this result is given in [14]; for our purposes, the following version is sufficient:

2.1. Proposition ([14]). Let $k(x, y) \in L^{1}\left(\mathbb{R}^{n} \times \mathbb{R}^{n}\right)$ satisfy the standard estimates

$$
|k(x, y)| \leq \kappa|x-y|^{-n}, \quad\left|\nabla_{x} k(x, y)\right|+\left|\nabla_{y} k(x, y)\right| \leq \kappa|x-y|^{-n-1} .
$$

Assume, moreover, that $T$ is bounded on $L^{2}\left(\mathbb{R}^{n}\right)$ with operator norm at most $\kappa$. Then $T$ is also bounded on $L^{p}\left(\mathbb{R}^{n}, X\right)$, where $X$ is any UMD space, with norm $\leq C_{p}(X) \kappa$, for all $\left.p \in\right] 1, \infty\left[\right.$, and it is bounded from $H^{1}\left(\mathbb{R}^{n}, X\right)$ to $L^{1}\left(\mathbb{R}^{n}, X\right)$ with norm $\leq C_{1}(X) \kappa$. If, in addition,

$$
\left[T^{\prime} 1\right](y):=\int_{\mathbb{R}^{n}} k(x, y) d x \equiv 0,
$$

then $T$ is bounded on $H^{1}\left(\mathbb{R}^{n}, X\right)$ with norm $\leq C_{0}(X) \kappa$.

This proposition is essentially a statement of the fact that for an operator defined in terms of a kernel which satisfies the standard estimates, the conditions of the $T 1$ theorem are necessary and sufficient: Since $T$ is bounded on $L^{2}\left(\mathbb{R}^{n}\right)$, it satisfies these conditions, but then the vector-valued version applies to give the boundedness on $L^{p}\left(\mathbb{R}^{n}, X\right)$. For our purposes, we would actually only need a special $T 1$ theorem, i.e., the case $T 1=0=T^{\prime} 1$.

It is a well known fact, in which the vector-valued situation brings no complications, that an integral operator satisfying the standard estimates and bounded on $L^{p}\left(\mathbb{R}^{n}, X\right)$ is also bounded from $H^{1}\left(\mathbb{R}^{n}, X\right)$ to $L^{1}\left(\mathbb{R}^{n}, X\right)$. As for the $H^{1}\left(\mathbb{R}^{n}, X\right)$-boundedness under the additional assumption, see Y. Meyer 
and R. Coifman [19, Th. 3 of Ch. 7]. (This is also an extension argument, which goes through in the vector-valued setting without modifications.)

2.2. Corollary. Let $\left(a_{\lambda}\right)_{\lambda \in \Lambda},\left(b_{\lambda}\right)_{\lambda \in \Lambda}$ be orthogonal sets in $L^{2}\left(\mathbb{R}^{n}\right)$ satisfying

$$
\left|a_{\lambda}(x)\right| \leq C_{m} \frac{2^{n j / 2}}{\left(1+\left|2^{j} x-k\right|\right)^{m}}, \quad\left|\nabla a_{\lambda}(x)\right| \leq C_{m} \frac{2^{n j / 2+j}}{\left(1+\left|2^{j} x-k\right|\right)^{m}}
$$

for all $\lambda=k 2^{-j}+\eta 2^{-j-1}$ and all $m \in \mathbb{N}$, with similar estimates for the $\left(b_{\lambda}\right)_{\lambda \in \Lambda}$. Consider the integral operators with kernels given by

$$
k(x, y)=\sum_{\lambda \in F} \nu_{\lambda} a_{\lambda}(x) b_{\lambda}(y)
$$

where $F \subset \Lambda$ is any finite set and $\nu_{\lambda} \in \mathbb{C},\left|\nu_{\lambda}\right| \leq 1$. These are uniformly bounded on $L^{p}\left(\mathbb{R}^{n}, X\right)$, and from $H^{1}\left(\mathbb{R}^{n}, X\right)$ to $L^{1}\left(\mathbb{R}^{n}, X\right)$, with the operator norms depending only on $p \in] 1, \infty[$, the UMD constant of the space $X$, and the quantities $C_{m}, m \in \mathbb{N}$. If the $a_{\lambda}$ 's have vanishing integral, then we also have boundedness on $H^{1}\left(\mathbb{R}^{n}, X\right)$ with a similar estimate for the norm.

Proof. From the assumed pointwise estimates, it easily follows that $\left\|a_{\lambda}\right\|_{2}$ $\leq C$, which depends only on the $C_{m}$ 's, and similarly $\left\|b_{\lambda}\right\|_{2} \leq C$. Then a bound depending only on the $C_{m}$ 's is easily derived for the operator norm of $f \mapsto \sum_{\lambda \in F} \nu_{\lambda} a_{\lambda}\left\langle b_{\lambda}, f\right\rangle$ on $L^{2}\left(\mathbb{R}^{n}\right)$, using the orthogonality of the two sets $\left(a_{\lambda}\right)$ and $\left(b_{\lambda}\right)$.

It is also a routine exercise to verify the standard estimates for the kernel $k$, with the constant only depending on the $C_{m}$ 's. Then the assertion follows from Prop. 2.1.

Now the first steps in our main theorem follow at once:

Proof of $(1.10) \Rightarrow(1.11) \Rightarrow(1.12)$. The first implication is immediate from the fact that, for any $F \subset \Lambda$ and $\varepsilon \in\{ \pm 1\}^{\Lambda}$,

$$
\sum_{\lambda \in F} \varepsilon_{\lambda} \psi_{\lambda}(x) \bar{\psi}_{\lambda}(y)
$$

are kernels of the kind considered in Cor. 2.2. Clearly the integral operator with the kernel given above maps $f$ to $\sum_{\lambda \in F} \varepsilon_{\lambda} \alpha_{\lambda} \psi_{\lambda}(\cdot)$.

The second implication is obvious, since the $L^{1}$ norm on the probability space $\Omega$ is dominated by the $L^{\infty}$ norm.

For the proof of further implications, we will need regular wavelet bases with the mother wavelet non-vanishing at a preassigned point. This is a somewhat untypical need, since usually it is the cancellation and vanishing properties of the wavelets which are desired.

2.3. LEMMA. For every $x \in \mathbb{R}$, there exists an infinitely regular wavelet $\psi$ on $\mathbb{R}$ such that $\psi(x) \neq 0$. 
Proof. The proof is based on a modification of Meyer's construction of the Littlewood-Paley multiresolution analysis $([18, \S 2.2])$, and the related wavelet $([18, \S 3.2])$. In that construction, one starts with an even, nonnegative function $\theta \in \mathcal{D}(\mathbb{R})$ such that $\theta(\xi)=1$ for $|\xi| \leq 2 \pi / 3, \theta(\xi)=0$ for $|\xi| \geq 4 \pi / 3$, and $\theta^{2}(\xi)+\theta^{2}(2 \pi-\xi)=1$ for $\xi \in[0,2 \pi]$. Our modification consists in choosing an $\eta \in C^{\infty}(\mathbb{R})$, which is required to be 0 on $[-2 \pi / 3,2 \pi / 3]$ but otherwise arbitrary, and taking $\vartheta(\xi):=\theta(\xi) e^{\mathbf{i} \eta(\xi)}$. We set $\phi:=\check{\vartheta}$, the inverse Fourier transform.

It follows, for $m(\xi):=\sum c_{k} e^{\mathrm{i} k \xi}$, that

$$
\begin{aligned}
\left\|\sum c_{k} \phi(x-k)\right\|_{2}^{2} & =\frac{1}{2 \pi}\|m(\xi) \vartheta(\xi)\|_{2}^{2}=\frac{1}{2 \pi} \sum_{j=-\infty}^{\infty} \int_{0}^{2 \pi}|m(\xi) \vartheta(\xi+2 \pi j)|^{2} d \xi \\
& =\frac{1}{2 \pi} \int_{0}^{2 \pi}|m(\xi)|^{2} d \xi=\sum\left|c_{k}\right|^{2}
\end{aligned}
$$

since $\sum|\vartheta(\xi+2 \pi j)|^{2} \equiv 1$, as is easily verified, and so $\phi(\cdot-k), k \in \mathbb{Z}$, are the orthonormal basis of a closed subspace $V_{0}$ of $L^{2}(\mathbb{R})$, which gives rise to a multiresolution analysis of $L^{2}(\mathbb{R})$.

We then pass to the construction of the corresponding wavelet $\psi$. Following [18, §3.2], we compute the auxiliary coefficients

$$
\alpha_{k}=\int_{-\infty}^{\infty} \frac{1}{2} \phi\left(\frac{x}{2}\right) \bar{\phi}(x+k) d x=\frac{1}{2 \pi} \int_{-\infty}^{\infty} \vartheta(2 \xi) \bar{\vartheta}(\xi) e^{\mathrm{i} k \xi} d \xi=\frac{1}{2} \phi\left(\frac{k}{2}\right),
$$

since $\vartheta(\xi)=1$ on the support of $\vartheta(2 \xi)$.

Then

$$
m_{0}(\xi):=\sum_{k=-\infty}^{\infty} \alpha_{k} e^{\mathbf{i} k \xi}=\sum_{k=-\infty}^{\infty} \vartheta(-2(\xi+2 k \pi))
$$

by Poisson's summation formula, and $\widehat{\psi}(\xi):=e^{-\mathbf{i} \xi / 2} \vartheta_{1}(\xi)$, where

$$
\vartheta_{1}(\xi):=\bar{m}_{0}(\xi / 2+\pi) \vartheta(\xi / 2)= \begin{cases}\vartheta(\xi / 2), & \xi \in \pm[4 \pi / 3,8 \pi / 3], \\ \bar{\vartheta}(-\xi \pm 2 \pi), & \xi \in \pm[2 \pi / 3,4 \pi / 3], \\ 0, & \text { else, }\end{cases}
$$

where the last equality follows readily upon taking into account the sets on which $\vartheta$ equals 1 or 0 . Note that $\left.\vartheta_{1}\right|_{ \pm[2 \pi / 3,4 \pi / 3]}$ is obtained from $\left.\vartheta_{1}\right|_{ \pm[4 \pi / 3,8 \pi / 3]}$ by reflecting and scaling about the point $\pm 4 \pi / 3$; in fact

$$
\begin{aligned}
\vartheta_{1}(4 \pi / 3-\xi) & =\bar{\vartheta}(2 \pi / 3+\xi), \\
\vartheta_{1}(4 \pi / 3+2 \xi) & =\vartheta(2 \pi / 3+\xi) \quad \text { for } \xi \in[0,2 \pi / 3],
\end{aligned}
$$


and similarly on the negative axis. Thus

$$
\begin{aligned}
\psi(x+1 / 2) & =\frac{1}{2 \pi} \int_{-\infty}^{\infty} e^{\mathbf{i} \xi(x+1 / 2)} e^{-\mathbf{i} \xi / 2} \vartheta_{1}(\xi) d \xi \\
= & \int_{0}^{2 \pi / 3}\left(\bar{\vartheta}(2 \pi / 3+\xi) e^{\mathbf{i}(4 \pi / 3-\xi) x}+2 \vartheta(2 \pi / 3+\xi) e^{\mathbf{i}(4 \pi / 3+2 \xi) x}\right) d \xi \\
& + \text { an integral over the negative half-line. }
\end{aligned}
$$

Now the phase of $\vartheta$ on $\pm[4 \pi / 3,8 \pi / 3]$ is under control; moreover, it can be adjusted independently on the positive and negative line segments. By symmetry, it then suffices to show that we can make the integral $\int_{0}^{2 \pi / 3}(\ldots) d \xi$ above non-vanishing by an appropriate choice of this phase. We choose this phase in such a way that

$$
\operatorname{Re} \int_{0}^{2 \pi / 3} \vartheta(2 \pi / 3+\xi) e^{\mathbf{i}(4 \pi / 3+2 \xi) x} d \xi \geq \frac{3}{4} \int_{0}^{2 \pi}|\vartheta(2 \pi / 3+\xi)| d \xi
$$

then the integral in (2.4) is estimated by

$$
\begin{aligned}
\left|\int_{0}^{2 \pi / 3}(I(\xi)+I I(\xi)) d \xi\right| & \geq\left|\int_{0}^{2 \pi / 3} I I(\xi) d \xi\right|-\int_{0}^{2 \pi / 3}|I(\xi)| d \xi \\
& \geq\left(\frac{3}{2}-1\right) \int_{0}^{2 \pi / 3}|\vartheta(2 \pi / 3+\xi)| d \xi>0
\end{aligned}
$$

Thus, for an arbitrary $x \in \mathbb{R}$, we have constructed a wavelet $\psi$ such that $\psi(x+1 / 2) \neq 0$; in fact, one with $|\psi(x+1 / 2)| \geq c$, where $c>0$ does not depend on $x$.

The $n$-dimensional version follows readily by a tensor product construction. Recall that the $2^{n}-1$ mother wavelets in the $n$-dimensional setting are naturally indexed by $\eta \in\{0,1\}^{n} \backslash\{0\}$. We denote by $\iota:=(1, \ldots, 1)$ the $n$-vector all of whose entries are 1.

2.5. Corollary. For any $x \in \mathbb{R}^{n}$, there exists an infinitely regular wavelet basis of $L^{2}\left(\mathbb{R}^{n}\right)$ such that $\psi^{\iota}(x) \neq 0$.

Proof. Let $\psi_{i, 0}:=\phi_{i}, \psi_{i, 1}:=\psi_{i}$ be (infinitely regular) father, resp. mother, wavelets on $\mathbb{R}$ for $i=1, \ldots, n$. For $\eta \in\{0,1\}^{n}, y \in \mathbb{R}^{n}$, define

$$
\psi^{\eta}(y):=\prod_{i=1}^{n} \psi_{i, \eta_{i}}\left(y_{i}\right)
$$

Then $\psi^{\eta}, \eta \in\{0,1\}^{n} \backslash\{0\}$, is the set of (infinitely regular) mother wavelets for a multiresolution analysis of $L^{2}\left(\mathbb{R}^{n}\right)$. By choosing the 1-dimensional wavelets 
$\psi_{i, 1}$ in such a way that $\psi_{i, 1}\left(x_{i}\right) \neq 0$ for a given $x=\left(x_{1}, \ldots, x_{n}\right)$, we clearly ensure the condition $\psi^{\iota}(x) \neq 0$.

Proof of $(1.10) \Rightarrow \forall A^{\eta}:(1.14) \Rightarrow(1.13)$. Let $A^{\eta}, \eta \in\{0,1\}^{n} \backslash\{0\}$, be non-degenerate cubes, and define

$$
A:=\bigcup_{\eta \in\{0,1\}^{n} \backslash\{0\}} \overline{A^{\eta}} ;
$$

this is a compact set.

For every $x \in A$, we choose an infinitely regular wavelet basis $\left(\psi_{x, \lambda}\right)_{\lambda \in \Lambda}$ such that $\psi_{x}^{\iota}(x) \neq 0$. By continuity of $\psi_{x}^{\iota}$, we have $\psi_{x}^{\iota}\left(U_{x}\right) \not \supset 0$ for some neighbourhood $U_{x}$ of $x$, and then by compactness we can choose finitely many, say $m$, infinitely regular wavelet bases $\left(\psi_{i, \lambda}\right)_{\lambda \in \Lambda}$ such that $\sum_{i=1}^{m}\left|\psi_{i}^{\iota}(x)\right| \geq$ $c>0$ for all $x \in A$. Now the kernels

$$
\sum_{\lambda \in F: \eta=\eta_{0}} \varepsilon_{\lambda} 2^{j n / 2} \psi_{i}^{\iota}\left(2^{j} x-k\right) \bar{\psi}_{\lambda}(y)
$$

satisfy the assumptions of Cor. 2.2; hence they define uniformly bounded integral operators from $H^{1}\left(\mathbb{R}^{n}, X\right)$ to $L^{1}\left(\mathbb{R}^{n}, X\right)$, and thus

$$
\sum_{i=1}^{m} E_{\varepsilon} \int_{\mathbb{R}^{n}}\left|\sum_{\lambda \in F} \varepsilon_{\lambda} \alpha_{\lambda} 2^{j n / 2} \psi_{i}^{\iota}\left(2^{j} x-k\right)\right|_{X} d x \leq C\|f\|_{H^{1}\left(\mathbb{R}^{n}, X\right)} .
$$

The contraction principle permits replacing $\psi_{i}^{\iota}\left(2^{j} x-k\right)$ by its absolute value above, and using the fact that $\sum_{i=1}^{m}\left|\psi_{i}^{\iota}\left(2^{j} x-k\right)\right| \geq c 1_{A^{\eta}}\left(2^{j} x-k\right)=c 1_{R(\lambda)}(x)$ and the contraction principle again, we finally deduce

$$
\left.\left.E_{\varepsilon} \int_{\mathbb{R}^{n}}\left|\sum_{\lambda \in F} \varepsilon_{\lambda} \alpha_{\lambda}\right| Q(\lambda)\right|^{-1 / 2} 1_{R(\lambda)}(x)\right|_{X} d x \leq C\|f\|_{H^{1}\left(\mathbb{R}^{n}, X\right)} .
$$

The fact that (1.14) for all $A^{\eta}$ implies (1.13) is evident, since (1.13) is just the special case of (1.14) with $A^{\eta}=\left[0,1\left[^{n}\right.\right.$.

Proof of (1.12) $\Rightarrow \exists A^{\eta}:$ (1.14). It suffices to observe that necessarily $\left|\psi^{\eta}(x)\right| \geq c>0$ for all $x$ in some cube $A^{\eta}$; then the expression in (1.14) can be dominated by that in (1.12) according to the contraction principle.

Now we have shown that

$$
\begin{aligned}
& (1.10) \Rightarrow(1.11) \Rightarrow(1.12) \Rightarrow \exists A^{\eta}:(1.14), \quad \text { and } \\
& (1.10) \Rightarrow \forall A^{\eta}:(1.14) \Rightarrow(1.13) \Rightarrow \exists A^{\eta}:(1.14)
\end{aligned}
$$

(where the last implication was not mentioned explicitly before, but it is trivial).

3. Construction of the atomic decomposition. To complete the proof of Theorem 1.9, we need to show that the condition (1.14), for any cubes $A^{\eta}$ whatsoever, implies the existence of an atomic decomposition for $f$; 
moreover, the $H^{1}$ norm of $f$ computed in terms of this decomposition should be controlled in terms of the expression in (1.14). Note that, without loss of generality, we may take the $A^{\eta}$ to be dyadic cubes of side-length $\leq 1$, since the expression in (1.14) decreases when the sets $A^{\eta}$ (and hence $R(\lambda)$ ) decrease. When this is done, it follows that the $R(\lambda)$ are dyadic cubes as well.

To achieve the atomic decomposition, we are going to modify the construction used by Meyer [18]. Certain parts of the proof are in almost oneto-one correspondence with the scalar-valued case; however, there are also significant departures from Meyer's reasoning.

Let us fix an $\eta_{0} \in\{0,1\}^{n} \backslash\{0\}$, and consider $f=\sum_{\lambda: \eta=\eta_{0}} \alpha_{\lambda} \psi_{\lambda}(x)$, where the summation runs over all $\lambda$ of the form $k 2^{-j}+\eta 2^{-j-1}$, where $\eta=\eta_{0}$. It clearly suffices to decompose each of the $2^{n}-1$ series of this kind. Then we can use a different indexing system which is more convenient in the present context: Let $\mathcal{R}$ be the collection of all the cubes $R(\lambda)=2^{-j}\left(A^{\eta}+k\right)$ such that $\eta=\eta_{0}$. Then, instead of $\Lambda$, we can use $\mathcal{R}$ as our index set, and we write $\varepsilon_{R}$ instead of $\varepsilon_{\lambda}$. Moreover, write $\alpha_{R}:=\alpha_{\lambda}$ for $R=R(\lambda)$ and $\eta=\eta_{0}$. Since $|Q(\lambda)|$ and $|R(\lambda)|$ only differ by a multiplicative constant independent of $\lambda$ (as long as $\eta=\eta_{0}$ is fixed), we can further replace the factor $|Q(\lambda)|^{-1 / 2}$ in our equations by $|R|^{-1 / 2}$.

Following [18], we set

$$
\sigma(x):=\left.\left.E_{\varepsilon}\left|\sum_{R \in \mathcal{R}} \varepsilon_{R} \alpha_{R}\right| R\right|^{-1 / 2} 1_{R}(x)\right|_{X} ;
$$

then $\sigma \in L^{1}\left(\mathbb{R}^{n}\right)$ by the standing assumption (1.14).

We further adopt the following notations:

$$
\begin{aligned}
& E_{k}:=\left\{x: \sigma(x)>2^{k}\right\}, \quad \mathcal{C}_{k}:=\left\{R \in \mathcal{R}:\left|R \cap E_{k}\right| \geq \beta|R|\right\}, \\
& \Delta_{k}:=\mathcal{C}_{k} \backslash \mathcal{C}_{k+1},
\end{aligned}
$$

where we fix some $\beta \in] 0,1\left[\right.$. Note that, if $\alpha_{R} \neq 0$, then $\sigma(x) \geq\left|\alpha_{R}\right|_{X}$ for all $x \in R$. Thus $R \subset E_{k}$ and hence $R \in \mathcal{C}_{k}$ for all small enough $k$.

The maximal members of $\mathcal{C}_{k}$ will be denoted by $R(k, l)$, where $l$ runs over an appropriate index set, and

$$
\Delta(k, l):=\left\{R \in \Delta_{k}: R \subset R(k, l)\right\} .
$$

Note that

$$
\sum_{l}|R(k, l)| \leq \sum_{l} \beta^{-1}\left|R(k, l) \cap E_{k}\right| \leq \beta^{-1}\left|E_{k}\right|
$$

and

$$
\sum_{k=-\infty}^{\infty} 2^{k}\left|E_{k}\right| \leq 2\|\sigma\|_{L^{1}\left(\mathbb{R}^{n}\right)} .
$$


We then come to a key estimate in the proof of $(1.14) \Rightarrow(1.10)$. The statement of this estimate is little more than a vector-valued analogue of the corresponding step in [18]; however, the proof is substantially longer and very different in spirit. The proof in [18] (where $p=2$ ) exploits the Hilbert space structure of the scalar-valued $L^{2}$ space, which at first seems to give little hope of extending the result beyond the Hilbert space framework. In view of this, it is perhaps surprising that the argument given below actually requires no geometric restrictions on the underlying Banach space $X$. The proof is very local in spirit; it essentially involves going through every cube $R \in \mathcal{R}$ one by one, in sharp contrast to the "global" argument in [18] in terms of the orthogonal expansions.

3.3. LEMMA. With the notation adopted above, we have the estimate

$$
\begin{aligned}
& \left.\left.\int_{\mathbb{R}^{n}} E_{\varepsilon}\left|\sum_{R \in \Delta(k, l)} \varepsilon_{R} \alpha_{R}\right| R\right|^{-1 / 2} 1_{R}(x)\right|_{X} ^{p} d x \\
& \leq\left.\left.\frac{1}{1-\beta} \int_{R(k, l) \backslash E_{k+1}} E_{\varepsilon}\left|\sum_{R \in \Delta(k, l)} \varepsilon_{R} \alpha_{R}\right| R\right|^{-1 / 2} 1_{R}(x)\right|_{X} ^{p} d x \leq c_{p} \frac{2^{(k+1) p}}{1-\beta}|R(k, l)| .
\end{aligned}
$$

Proof. The second inequality is clear from Kahane's inequality $E_{\varepsilon}\left|\sum \varepsilon_{i} x_{i}\right|_{X}^{p} \leq c_{p}\left(E_{\varepsilon}\left|\sum \varepsilon_{i} x_{i}\right|_{X}\right)^{p}$ and the fact that $\sigma(x) \leq 2^{k+1}$ for $x \notin$ $E_{k+1}$. Therefore we will concentrate on the first inequality.

Observe that if $R_{1} \cap R_{2} \neq \emptyset$, then necessarily $R_{1} \subset R_{2}$ or $R_{2} \subset R_{1}$, since $R_{1}, R_{2}$ are dyadic cubes. If $\widetilde{R} \in \Delta(k, l)$ is minimal, in the sense that $R \subsetneq \widetilde{R} \Rightarrow R \notin \Delta(k, l)$, then for $x \in \widetilde{R}$ we have

$$
\left.\left.E_{\varepsilon}\left|\sum_{R \in \Delta(k, l)} \varepsilon_{R} \alpha_{R}\right| R\right|^{-1 / 2} 1_{R}(x)\right|_{X}=\left.\left.E_{\varepsilon}\left|\sum_{R \in \Delta(k, l), R \supset \widetilde{R}} \varepsilon_{R} \alpha_{R}\right| R\right|^{-1 / 2}\right|_{X}
$$

i.e., this expression is constant for $x \in \widetilde{R}$.

More generally, if $\widetilde{R} \in \Delta(k, l)$, and

$$
\widetilde{R}_{0}:=\widetilde{R} \backslash \bigcup_{\substack{R \in \Delta(k, l) \\ R \subsetneq \widetilde{R}}} R
$$

then (3.4) holds for all $x \in \widetilde{R}_{0}$.

It suffices to establish the assertion of the lemma in the case when only finitely many $\alpha(Q)$ are non-zero, since the general case then follows from the monotone convergence theorem. Then the summations involved are finite, and we can avoid all convergence problems in the following. Replacing $\Delta(k, l)$ by $\left\{R \in \Delta(k, l): \alpha_{R} \neq 0\right\}$ if necessary, we can assume that $\Delta(k, l)$ is finite.

Let $R$ be one of the maximal members of $\Delta(k, l)$. It clearly suffices to prove, for all such $R$, that 


$$
\begin{aligned}
\int_{R} E_{\varepsilon} \mid & \left.\sum_{\widetilde{R} \in \Delta(k, l), \widetilde{R} \subset R} \varepsilon_{\widetilde{R}} \alpha_{\widetilde{R}}|\widetilde{R}|^{-1 / 2} 1_{\widetilde{R}}(x)\right|_{X} ^{p} d x \\
& \leq\left.\left.\frac{1}{1-\beta} \int_{R \backslash E_{k+1}} E_{\varepsilon}\right|_{\widetilde{R} \in \Delta(k, l), \widetilde{R} \subset R} \sum_{\widetilde{R}} \alpha_{\widetilde{R}}|\widetilde{R}|^{-1 / 2} 1_{\widetilde{R}}(x)\right|_{X} ^{p} d x .
\end{aligned}
$$

To prove this inequality, we need to introduce some notation. We say that $\widetilde{R}$ is a $\Delta$-subcube of $R$ if $\widetilde{R} \subsetneq R$ and $\widetilde{R} \in \Delta(k, l)$. We say that $\widetilde{R}$ is a first order $\Delta$-subcube of $R$ if, in addition, the following property holds: there is no $\widehat{R} \in \Delta(k, l)$ with $\widetilde{R} \subsetneq \widehat{R} \subsetneq R$. We label the first order $\Delta$-subcubes of $R$ by $R_{i}$, where $i$ runs over an appropriate finite index set. The first order $\Delta$-subcubes of $R_{i}$, which are labelled $R_{i j}$, are called the second order $\Delta$-subcubes of $R$, and so on. The $m$ th order $\Delta$-subcubes of $R$ will be denoted by $R_{\alpha}$, where $\alpha=\alpha_{1} \ldots \alpha_{m}$ is a string of $m$ indices. We further define $R_{\alpha 0}:=R_{\alpha} \backslash \bigcup R_{\alpha i}$, which is obviously equivalent to the earlier definition (3.5). For convenience, we also set $E:=E_{k+1}$.

Since the proof of the inequality (3.6) in the general situation involves a large amount of indices, it is helpful to consider first a special case in which only first and second order $\Delta$-subcubes of $R$ are involved. If $S \subset R$, we denote by $I(S)$ the integral over $S$ of the same integrand as in (3.6), and $\mu(S):=I(S) /|S|$ if $|S|>0$, and $\mu(S):=0$ otherwise.

Now in our special situation, the cube $R$ is decomposed into disjoint parts as follows:

$$
R=R_{0} \cup \bigcup_{i \in I} R_{i} \cup \bigcup_{j \in J}\left(R_{j 0} \cup \bigcup_{k \in K_{j}} R_{j k}\right),
$$

where $R_{i}, i \in I$, are those first order $\Delta$-subcubes of $R$ which have no further $\Delta$-subcubes, whereas $R_{j}=\bigcup_{k \in\{0\} \cup K_{j}} R_{j k}, j \in J$, are those first order $\Delta$ subcubes of $R$ which do have some further $\Delta$-subcubes, namely the $R_{j k}$, $k \in K_{j}$.

Now

$$
\begin{aligned}
I(R \backslash E)= & I\left(R_{0} \backslash E\right)+\sum_{i \in I} I\left(R_{i} \backslash E\right)+\sum_{j \in J}\left(I\left(R_{j 0} \backslash E\right)+\sum_{k \in K_{j}} I\left(R_{j k} \backslash E\right)\right) \\
= & \left|R_{0} \backslash E\right| \mu\left(R_{0}\right)+\sum_{i \in I}\left|R_{i} \backslash E\right| \mu\left(R_{i}\right) \\
& +\sum_{j \in J}\left(\left|R_{j 0} \backslash E\right| \mu\left(R_{j 0}\right)+\sum_{k \in K_{j}}\left|R_{j k} \backslash E\right| \mu\left(R_{j k}\right)\right),
\end{aligned}
$$

since the integrand is constant on each of the sets $R_{0}, R_{i}, R_{j 0}, R_{j k}$, as was observed above.

We want to show that the above displayed quantity is at least $(1-\beta) I(R)$ $=: t I(R)$, where $t:=1-\beta$. To see this, observe that $|\bar{R} \cap E|=\left|\bar{R} \cap E_{k+1}\right|<$ 
$\beta|\bar{R}|$, hence $|\bar{R} \backslash E|>(1-\beta)|\bar{R}|$ for all $\bar{R} \in \Delta_{k} \subset \mathcal{C}_{k+1}^{\mathrm{c}}$ by the definition of $\mathcal{C}_{k+1}$. Now

$$
t I(R)=t\left|R_{0}\right| \mu\left(R_{0}\right)+\sum_{i \in I} t\left|R_{i}\right| \mu\left(R_{i}\right)+\sum_{j \in J}\left(t\left|R_{j 0}\right| \mu\left(R_{j 0}\right)+\sum_{k \in K_{j}} t\left|R_{j k}\right| \mu\left(R_{j k}\right)\right),
$$

and hence

$$
\begin{aligned}
& I(R \backslash E)-t I(R)=\left(\left|R_{0} \backslash E\right|-t\left|R_{0}\right|\right) \mu\left(R_{0}\right)+\sum_{i \in I}\left(\left|R_{i} \backslash E\right|-t\left|R_{i}\right|\right) \mu\left(R_{i}\right) \\
& \quad+\sum_{j \in J}\left[\left(\left|R_{j 0} \backslash E\right|-t\left|R_{j 0}\right|\right) \mu\left(R_{j 0}\right)+\sum_{k \in K_{j}}\left(\left|R_{j k} \backslash E\right|-t\left|R_{j k}\right|\right) \mu\left(R_{j k}\right)\right],
\end{aligned}
$$

and if we set $\tau(S):=|S \backslash E|-t|S|$ (whence $\tau(\bar{R})>0$ for all $\bar{R} \in \Delta_{k}$ ), this can be further written as

$$
\begin{aligned}
=[ & \left.\tau\left(R_{0}\right)+\sum_{i \in I} \tau\left(R_{i}\right)+\sum_{j \in J} \sum_{k \in\{0\} \cup K_{j}} \tau\left(R_{j k}\right)\right] \mu\left(R_{0}\right) \\
+\sum_{i \in I} \tau\left(R_{i}\right)\left(\mu\left(R_{i}\right)-\mu\left(R_{0}\right)\right)+\sum_{j \in J}( & \left\{\sum_{k \in\{0\} \cup K_{j}} \tau\left(R_{j k}\right)\right\}\left(\mu\left(R_{j 0}\right)-\mu\left(R_{0}\right)\right) \\
& \left.+\sum_{k \in K_{j}} \tau\left(R_{j k}\right)\left(\mu\left(R_{j k}\right)-\mu\left(R_{j 0}\right)\right)\right) .
\end{aligned}
$$

Noting that the quantity in brackets $[\cdots]$ is simply $\tau(R)$, whereas that in braces $\{\cdots\}$ is $\tau\left(R_{j}\right)$, we find that all the terms appearing above are nonnegative, and hence $I(R \backslash E) \geq t I(R)$, which we wanted to prove.

The special case treated above already contains the essence of the matter, and it is essentially the notation which is more difficult in the general case where $\Delta$-subcubes of higher orders are allowed. Now $R$ is disjointly decomposed as

$$
R=R_{0} \cup \bigcup_{\alpha}\left(\bigcup_{i} R_{\alpha i} \cup \bigcup_{j} R_{\alpha j 0}\right),
$$

where $\alpha$ runs over an appropriate set of strings of indices, and $i$ and $j$ over appropriate sets (possibly different for different $\alpha$ ) of single indices. Note that the possibility of $\alpha$ being the empty string is allowed. The decomposition (3.8) should be compared with the special case in (3.7).

We have

$$
\begin{aligned}
& I(R \backslash E)-t I(R)=\left(\left|R_{0} \backslash E\right|-t\left|R_{0}\right|\right) \mu\left(R_{0}\right) \\
& +\sum_{\alpha}\left[\sum_{i}\left(\left|R_{\alpha i} \backslash E\right|-t\left|R_{\alpha i}\right|\right) \mu\left(R_{\alpha i}\right)+\sum_{j}\left(\left|R_{\alpha j 0} \backslash E\right|-t\left|R_{\alpha j 0}\right|\right) \mu\left(R_{\alpha j 0}\right)\right] \\
& \quad=\tau\left(R_{0}\right) \mu\left(R_{0}\right)+\sum_{\alpha}\left(\sum_{i} \tau\left(R_{\alpha i}\right) \mu\left(R_{\alpha i}\right)+\sum_{j} \tau\left(R_{\alpha j 0}\right) \mu\left(R_{\alpha j 0}\right)\right) .
\end{aligned}
$$


We claim that this is equal to

$$
\begin{aligned}
\left\{\tau\left(R_{0}\right)+\sum_{\alpha}\left(\sum_{i} \tau\left(R_{\alpha i}\right)+\sum_{j} \tau\left(R_{\alpha j 0}\right)\right)\right\} \mu\left(R_{0}\right) & \\
& +\sum_{\alpha} \sum_{i} \tau\left(R_{\alpha i}\right)\left(\mu\left(R_{\alpha i}\right)-\mu\left(R_{\alpha 0}\right)\right) \\
+\sum_{\alpha} \sum_{j}\left[\tau\left(R_{\alpha j 0}\right)+\sum_{\beta}\left(\sum_{k} \tau\left(R_{\alpha j \beta k}\right)\right.\right. & \left.\left.+\sum_{l} \tau\left(R_{\alpha j \beta l 0}\right)\right)\right]\left(\mu\left(R_{\alpha j 0}\right)-\mu\left(R_{\alpha 0}\right)\right) .
\end{aligned}
$$

In the expression above, the quantity in braces $\{\cdots\}$ is $\tau(R) \geq 0$ and that in brackets $[\cdots]$ is $\tau\left(R_{\alpha j}\right) \geq 0$, so that all the terms appearing above are nonnegative. Hence it suffices to verify the claimed equality, i.e., the vanishing of the expression

$$
\begin{aligned}
\sum_{\alpha, i} \tau\left(R_{\alpha i}\right) \mu\left(R_{0}\right)+ & \sum_{\alpha, j} \tau\left(R_{\alpha j 0}\right) \mu\left(R_{0}\right) \\
& -\sum_{\alpha, i} \tau\left(R_{\alpha i}\right) \mu\left(R_{\alpha 0}\right)-\sum_{\alpha, j} \tau\left(R_{\alpha j 0}\right) \mu\left(R_{\alpha 0}\right) \\
& +\sum_{\alpha, j, \beta}\left(\sum_{k} \tau\left(R_{\alpha j \beta k}\right)+\sum_{l} \tau\left(R_{\alpha j \beta l 0}\right)\right) \mu\left(R_{\alpha j 0}\right) \\
& -\sum_{\alpha, j, \beta}\left(\sum_{k} \tau\left(R_{\alpha j \beta k}\right)+\sum_{l} \tau\left(R_{\alpha j \beta l 0}\right)\right) \mu\left(R_{\alpha 0}\right) .
\end{aligned}
$$

When $\alpha$ runs over all strings, and $j$ over all single indices, $\alpha j$ clearly runs over all strings except for the empty string. Hence the second-to-last term in $(3.9)$ is equal to

$$
\begin{aligned}
\sum_{\alpha, \beta}\left[\sum_{k} \tau\left(R_{\alpha \beta k}\right)+\sum_{l} \tau\left(R_{\alpha \beta l 0}\right)\right] \mu\left(R_{\alpha 0}\right) & \\
& -\sum_{\beta, k} \tau\left(R_{\beta k}\right) \mu\left(R_{0}\right)-\sum_{\beta, l} \tau\left(R_{\beta l 0}\right) \mu\left(R_{0}\right) .
\end{aligned}
$$

Similarly, replacing the pair $(j, \beta)$ by $\beta$ alone in the last term of $(3.9)$, we find that this last term is equal to

$$
\begin{aligned}
-\sum_{\alpha, \beta}\left[\sum_{k} \tau\left(R_{\alpha \beta k}\right)+\sum_{l} \tau\left(R_{\alpha \beta l 0}\right)\right] \mu\left(R_{\alpha 0}\right) & +\sum_{\alpha, k} \tau\left(R_{\alpha k}\right) \mu\left(R_{\alpha 0}\right) \\
& +\sum_{\alpha, l} \tau\left(R_{\alpha l 0}\right) \mu\left(R_{\alpha 0}\right) .
\end{aligned}
$$

Now it is clear that the different terms in (3.9) cancel each other, so our claim, and hence the assertion of the lemma, is verified. 
Now we define

$$
A_{k, l}(x, \varepsilon):=\sum_{R \in \Delta(k, l)} \varepsilon_{R} \alpha_{R}|R|^{-1 / 2} 1_{R}(x)
$$

note that

$$
\sum_{k=-\infty}^{\infty} \sum_{l} A_{k, l}(x, \varepsilon)=\sum_{R \in \mathcal{R}} \varepsilon_{R} \alpha_{R}|R|^{-1 / 2} 1_{R}(x) .
$$

A modification of this series will give us the required atomic decomposition of $f$. Observe that $\operatorname{supp} A_{k, l}(\cdot, \varepsilon) \subset R(k, l)$ by the definition of $\Delta(k, l)$.

Moreover, by Lemma 3.3, we have

$$
\begin{aligned}
\sum_{k, l}\left\|A_{k, l}\right\|_{L^{p}\left(\Omega \times \mathbb{R}^{n}, X\right)}|R(k, l)|^{1 / p^{\prime}} & \\
& \leq \sum_{k, l} c_{p}^{1 / p}(1-\beta)^{-1 / p} 2^{k+1}|R(k, l)|^{1 / p}|R(k, l)|^{1 / p^{\prime}} \\
& \leq 2 c_{p}^{1 / p}(1-\beta)^{-1 / p} \sum_{k} 2^{k} \sum_{l}|R(k, l)| \\
& \stackrel{(3.1)}{\leq} 2 c_{p}^{1 / p}(1-\beta)^{-1 / p} \beta^{-1} \sum_{k} 2^{k}\left|E_{k}\right| \\
& \stackrel{(3.2)}{\leq} 4 c_{p}^{1 / p}(1-\beta)^{-1 / p} \beta^{-1}\|\sigma\|_{L^{1}\left(\mathbb{R}^{n}\right)} .
\end{aligned}
$$

The quantity on the left of this estimate should be compared with the definition of the $H^{1}$ norm in (1.1).

Now we are ready to finish the proof of Theorem 1.9.

Conclusion of the proof of $(1.14) \Rightarrow(1.10)$. Now we construct the atomic decomposition of $f$, or more precisely, of each of the subseries

$$
f_{\eta_{0}}(x):=\sum_{\lambda \in \Lambda: \eta=\eta_{0}} \alpha_{\lambda} \psi_{\lambda}(x)=\sum_{R \in \mathcal{R}} \alpha_{R} \psi_{\lambda(R)}(x)
$$

where $\lambda(R):=2^{-j} k+2^{-j-1} \eta_{0}$ for $R=2^{-j}\left(A^{\eta_{0}}+k\right)$.

Consider a basis $\left(\Psi_{\lambda}\right)_{\lambda \in \Lambda}$ of compactly supported, 1-regular wavelets. The existence of such wavelet bases is well known ([18]). Now that $A^{\eta_{0}}$ is a nondegenerate cube, we have supp $\Psi_{2^{-j_{0}} k_{0}+2^{-j_{0}-1} \eta_{0}}=\operatorname{supp} 2^{j_{0} n / 2} \Psi^{\eta_{0}}\left(2^{j_{0}} \cdot-k_{0}\right) \subset$ $A^{\eta_{0}}$ for some suitable $j_{0} \geq 0$ and $k_{0} \in \mathbb{Z}^{n}$.

Define $\Psi_{j, k}^{\eta}:=\Psi_{\lambda}$ for $\lambda=2^{-j} k+2^{-j-1} \eta$, and set $\phi:=\Psi_{j_{0}, k_{0}}^{\eta_{0}}$, and

$$
\phi_{j, k}:=2^{n j / 2} \phi\left(2^{j} \cdot-k\right)=2^{n\left(j+j_{0}\right) / 2} \Psi^{\eta_{0}}\left(2^{j_{0}}\left(2^{j} \cdot-k\right)-k_{0}\right)=\Psi_{j+j_{0}, 2^{j_{0}} k+k_{0}}^{\eta_{0}} .
$$

Since $j_{0} \geq 0$, we see that $\left(\phi_{j, k}\right)_{j \in \mathbb{Z}, k \in \mathbb{Z}^{n}}$ is a subset of $\left(\psi_{\lambda}\right)_{\lambda \in \Lambda}$, thus orthonormal (but not complete, of course) in $L^{2}\left(\mathbb{R}^{n}\right)$. 
Now that $\phi$ is bounded and supported on $A^{\eta_{0}}$, we have

$$
|\phi(x)| \leq C\left|A^{\eta_{0}}\right|^{-1 / 2} 1_{A^{\eta_{0}}}(x),
$$

where $C=\|\phi\|_{\infty}\left|A^{\eta_{0}}\right|^{1 / 2}$, and then by scaling

$$
\left|\phi_{R}(x)\right|:=\left|\phi_{j, k}(x)\right| \leq C|R|^{-1 / 2} 1_{R}(x)
$$

for $R=2^{-j}\left(A^{\eta_{0}}+k\right)$. Then the contraction principle gives

$$
\int_{\mathbb{R}^{n}} E_{\varepsilon}\left|\sum_{R \in \Delta(k, l)} \varepsilon_{R} \alpha_{R} \phi_{R}(x)\right|_{X}^{p} d x \leq\left.\left. C \int_{\mathbb{R}^{n}} E_{\varepsilon}\left|\sum_{R \in \Delta(k, l)} \varepsilon_{R} \alpha_{R}\right| R\right|^{-1 / 2} 1_{R}(x)\right|_{X} ^{p} d x .
$$

Now we apply Cor. 2.2 with

$$
\sum_{\lambda \in \Lambda: \eta=\eta_{0}} \varepsilon_{R(\lambda)} \psi_{\lambda}(x) \bar{\phi}_{R(\lambda)}(y)
$$

to get

$$
\int_{\mathbb{R}^{n}}\left|\sum_{\substack{\lambda \in \Lambda: \eta=\eta_{0}, R(\lambda) \in \Delta(k, l)}} \alpha_{\lambda} \psi_{\lambda}(x)\right|_{X}^{p} d x \leq\left.\left.\int_{\mathbb{R}^{n}}\left|\sum_{R \in \Delta(k, l)} \varepsilon_{R} \alpha_{R}\right| R\right|^{-1 / 2} 1_{R}(x)\right|_{X} ^{p} d x
$$

Taking the expectation $E_{\varepsilon}$ of the right-hand side and combining this with the previous inequality, we have shown, for

$$
a_{k, l}(x):=\sum_{\substack{\lambda \in \Lambda: \eta=\eta_{0}, R(\lambda) \in \Delta(k, l)}} \alpha_{\lambda} \psi_{\lambda}(x),
$$

the estimate

$$
\left\|a_{k, l}\right\|_{L^{p}\left(\mathbb{R}^{n}, X\right)} \leq C\left\|A_{k, l}\right\|_{L^{p}\left(\Omega \times \mathbb{R}^{n}, X\right)} .
$$

Since each of the wavelets $\psi_{\lambda}$ has a vanishing integral, so does $a_{k, l}$. Consider two cases:

The case of compactly supported wavelets. Since $A^{\eta}$ is a non-degenerate cube and $\psi^{\eta}$ has compact support, we have $\operatorname{supp} \psi^{\eta} \subset\left(A^{\eta}\right)^{*}$ where $Q^{*}$ denotes the cube concentric with $Q$ and having $g$ times the side length of $Q$, where $g$ is a sufficiently large constant. Then $\psi_{j, k}^{\eta}=\psi_{2^{-j} k+2^{-j-1} \eta}=$ $2^{j n / 2} \psi^{\eta}\left(2^{j} \cdot-k\right)$ satisfies $\operatorname{supp} \psi_{j, k}^{\eta}=2^{-j}\left(\operatorname{supp} \psi^{\eta}+k\right) \subset 2^{-j}\left(\left(A^{\eta}\right)^{*}+k\right)=$ $\left(2^{-j}\left(A^{\eta}+k\right)\right)^{*}$, i.e., $\operatorname{supp} \psi_{\lambda} \subset R(\lambda)^{*}$.

Thus, if $R(\lambda) \in \Delta(k, l)$, hence $R(\lambda) \subset R(k, l)$, we have supp $\psi_{\lambda} \subset$ $R(k, l)^{*}$. This means that $\operatorname{supp} a_{k, l} \subset R(k, l)^{*}$, and then

$$
\begin{aligned}
\left\|f_{\eta_{0}}\right\|_{H^{1}\left(\mathbb{R}^{n}, X\right)} & \leq \sum_{k, l}\left\|a_{k, l}\right\|_{L^{p}\left(\mathbb{R}^{n}, X\right)}\left|R(k, l)^{*}\right|^{1 / p^{\prime}} \\
& \stackrel{(3.11)}{\leq} C \sum_{k, l}\left\|A_{k, l}\right\|_{L^{p}\left(\Omega \times \mathbb{R}^{n}, X\right)}|R(k, l)|^{1 / p^{\prime}} \stackrel{(3.10)}{\leq} C\|\sigma\|_{L^{1}\left(\mathbb{R}^{n}\right)} .
\end{aligned}
$$


Thus we obtain a norm estimate for $f_{\eta_{0}}$, and then for $f=\sum_{\eta \in\{0,1\}^{n} \backslash\{0\}} f_{\eta}$, of the desired form.

The general case. By the special case considered above, we obtain

$$
\left\|\sum_{\lambda \in \Lambda} \alpha_{\lambda} \Psi_{\lambda}\right\|_{H^{1}\left(\mathbb{R}^{n}, X\right)} \leq C\|\sigma\|_{L^{1}\left(\mathbb{R}^{n}\right)},
$$

where $\left(\Psi_{\lambda}\right)_{\lambda \in \Lambda}$ is a compactly supported 1-regular wavelet basis as above. Then it suffices to apply the $H^{1}\left(\mathbb{R}^{n}, X\right)$-boundedness assertion of Cor. 2.2 to

$$
\sum \psi_{\lambda}(x) \bar{\Psi}_{\lambda}(y)
$$

to deduce the desired norm estimate for $f=\sum \alpha_{\lambda} \psi_{\lambda}$, where $\left(\psi_{\lambda}\right)_{\lambda \in \Lambda}$ is any 1-regular wavelet basis. This completes the proof of $(1.14) \Rightarrow(1.10)$, and of Theorem 1.9.

4. On $\operatorname{BMO}\left(\mathbb{R}^{n}, X\right)$ and duality. One can also generalize the wavelet characterization of the space $\operatorname{BMO}\left(\mathbb{R}^{n}\right)$ from [18] to the UMD-valued situation. This generalization is not as exciting as that of the characterization of $H^{1}\left(\mathbb{R}^{n}\right)$; in essence, we just need to replace classical $L^{2}$ estimates used in [18] by the application of Cor. 2.2, but otherwise the proof follows the lines of [18].

4.1. Proposition. Let $X$ be a UMD space and $\left(\psi_{\lambda}\right)_{\lambda \in \Lambda}$ a 1-regular wavelet basis. If $\left.b \in \operatorname{BMO}\left(\mathbb{R}^{n}, X\right), p \in\right] 1, \infty\left[\right.$ and $\alpha_{\lambda}:=\left\langle b, \bar{\psi}_{\lambda}\right\rangle$, then

$$
\int_{\mathbb{R}^{n}} E_{\varepsilon}\left|\sum_{\lambda \in F} \varepsilon_{\lambda} \alpha_{\lambda} \psi_{\lambda}(x)\right|_{X}^{p} d x \leq \kappa^{p}|Q| \quad \forall F \subset\{\lambda \in \Lambda: Q(\lambda) \subset Q\},
$$

where $\kappa \leq C_{p}\|b\|_{\mathrm{BMO}\left(\mathbb{R}^{n}, X\right)}$.

Conversely, if (4.2) holds for some set of coefficients $\left(\alpha_{\lambda}\right)_{\lambda \in \Lambda} \subset X$ and all finite sets $F$ as above, then the series

$$
\sum_{\lambda \in \Lambda} \alpha_{\lambda} \psi_{\lambda}(x)
$$

converges unconditionally in $L_{\mathrm{loc}}^{p}\left(\mathbb{R}^{n}, X\right) / X$ to a function in $\operatorname{BMO}\left(\mathbb{R}^{n}, X\right)$ with norm at most $C_{p} \kappa$.

By convergence in $L_{\mathrm{loc}}^{p}\left(\mathbb{R}^{n}, X\right) / X$ we mean the following: For every compact $K \subset \mathbb{R}^{n}$, there exist "renormalization constants" $c_{\lambda} \in X$ such that $\sum_{\lambda \in \Lambda}\left(\alpha_{\lambda} \psi_{\lambda}(\cdot)+c_{\lambda}\right)$ converges in $L^{p}(K, X)$.

Proof. We give the proof in the case of compactly supported wavelets, since the additional considerations required by the general case do not involve the vector-valuedness of the functions in any way. The required modifications are left as an exercise for the reader in Meyer's book [18], and we follow him here. Thus, under the additional assumption, we have $\operatorname{supp} \psi_{\lambda} \subset Q(\lambda)^{*}$. 
Necessity of (4.2). Writing

$$
b:=\left(b-b_{Q^{*}}\right) 1_{Q^{*}}+\left(b-b_{Q^{*}}\right) 1_{\left(Q^{*}\right)^{c}}+b_{Q^{*}}=: b_{1}+b_{2}+b_{3},
$$

where $b_{Q^{*}}:=\left|Q^{*}\right|^{-1} \int_{Q^{*}} b(x) d x$, we find that $\left\langle b_{2}, \bar{\psi}_{\lambda}\right\rangle=0$ if $Q(\lambda) \subset Q$ (since then $\left.\operatorname{supp} \psi_{\lambda} \subset Q^{*}\right)$, and $\left\langle b_{3}, \bar{\psi}_{\lambda}\right\rangle=0$ for all $\lambda \in \Lambda$, since $\int \psi_{\lambda}(x) d x=0$. Thus, when $Q(\lambda) \subset Q$, we have

$$
\alpha_{\lambda}=\left\langle b, \bar{\psi}_{\lambda}\right\rangle=\left\langle\left(b-b_{Q^{*}}\right) 1_{Q^{*}}, \bar{\psi}_{\lambda}\right\rangle
$$

and so

$$
\begin{aligned}
\int_{\mathbb{R}^{n}} E_{\varepsilon}\left|\sum_{Q(\lambda) \subset Q} \varepsilon_{\lambda} \alpha_{\lambda} \psi_{\lambda}(x)\right|_{X}^{p} d x & \leq C\left\|\left(b-b_{Q^{*}}\right) 1_{Q^{*}}\right\|_{L^{p}\left(\mathbb{R}^{n}, X\right)}^{p} \\
& \leq C\left|Q^{*}\right|\|b\|_{\mathrm{BMO}\left(\mathbb{R}^{n}, X\right)}^{p} .
\end{aligned}
$$

This completes the first half of the proof.

Sufficiency of (4.2). Let $\bar{B}$ be a ball of radius $r$. We investigate separately the two series

$$
\sum_{|Q(\lambda)| \leq|\bar{B}|} \alpha_{\lambda} \psi_{\lambda}(x) \text { and } \sum_{|Q(\lambda)|>|\bar{B}|} \alpha_{\lambda} \psi_{\lambda}(x) .
$$

Concerning the first series, if $x \in \bar{B}$ and $x \in \operatorname{supp} \psi_{\lambda} \subset Q(\lambda)^{*}$ for some $x$, then $\bar{B} \cap Q(\lambda)^{*} \neq \emptyset$, and from the size assumption $|Q(\lambda)| \leq|\bar{B}|$ it follows that $Q(\lambda) \subset \bar{B}^{\star}$, where the $\star$ designates expansion about the same centre by a sufficiently large factor which only depends on the expansion factor implicit in the notation $Q(\lambda)^{*}$. Thus

$$
\begin{aligned}
\int_{\mathbb{R}^{n}} E_{\varepsilon} \mid & \left.\sum_{\substack{\lambda \in F:|Q(\lambda)| \leq|\bar{B}|, \bar{B} \cap \operatorname{supp} \psi_{\lambda} \neq \emptyset}} \varepsilon_{\lambda} \alpha_{\lambda} \psi_{\lambda}(x)\right|_{X} ^{p} d x \\
& \leq \int_{\mathbb{R}^{n}} E_{\varepsilon}\left|\sum_{\lambda \in F: Q(\lambda) \subset \bar{B}^{\star}} \cdots\right|_{X}^{p} d x \leq c \kappa^{p}|\bar{B}| .
\end{aligned}
$$

From this estimate, which is uniform for finite sets $F \subset \Lambda$, and the fact that $c_{0} \not \subset X$ for $X \mathrm{UMD}$, it follows that the series $\sum \varepsilon_{\lambda} \alpha_{\lambda} \psi_{\lambda}(\cdot)$ (summation over $\lambda \in \Lambda$ with $|Q(\lambda)| \leq|\bar{B}|$ and $\bar{B} \cap \operatorname{supp} \psi_{\lambda} \neq \emptyset$ ) converges almost surely (with respect to the $\varepsilon_{\lambda}$ 's) in $L^{p}\left(\mathbb{R}^{n}, X\right)$. But due to the $L^{p}\left(\mathbb{R}^{n}, X\right)$ boundedness of the integral transformations with kernels $\sum \varepsilon_{\lambda} \psi_{\lambda}(x) \bar{\psi}_{\lambda}(y)$, it actually converges surely, i.e., $\sum \alpha_{\lambda} \psi_{\lambda}(x)$ (summation restricted as above) converges unconditionally. For $x \in \bar{B}$, this series agrees with

$$
\sum_{\lambda \in \Lambda,|Q(\lambda)| \leq|\bar{B}|} \alpha_{\lambda} \psi_{\lambda}(x)
$$

which hence converges unconditionally in $L^{p}(\bar{B}, X)$. 
We then consider summation over $|Q(\lambda)|>|\bar{B}|$. For each fixed size $2^{-j n}=|Q(\lambda)|$, there are at most a bounded number, say $m$, of dyadic cubes $Q(\lambda)$ such that $Q(\lambda)^{*} \cap \bar{B} \neq \emptyset$. Moreover, denoting by $x_{0}$ the centre of $\bar{B}$, we have, for $x \in \bar{B}$,

$$
\left|\psi_{\lambda}(x)-\psi_{\lambda}\left(x_{0}\right)\right| \leq\left|\left(x-x_{0}\right) \cdot \nabla \psi_{\lambda}(\xi)\right| \leq C 2^{n j / 2+j} r,
$$

where $r$ is the radius of $\bar{B}$ and $\lambda=2^{-j} k+2^{-j-1} \eta$. From (4.2) it follows that $\left|\alpha_{\lambda}\right|_{X} \leq C \kappa 2^{-n j / 2}$. Combining these observations yields

$$
\begin{aligned}
\sum_{|Q(\lambda)|>|\bar{B}|, Q(\lambda)^{*} \cap \bar{B} \neq \emptyset}\left|\alpha_{\lambda}\right| X\left|\psi_{\lambda}(x)-\psi_{\lambda}\left(x_{0}\right)\right| & \leq \sum_{2^{-j n}>|\bar{B}|} m \kappa 2^{-n j / 2} C 2^{n j / 2+j} r \leq c \kappa \sum_{2^{j}<r^{-1}} 2^{j} r \leq c \kappa,
\end{aligned}
$$

and this shows that $\sum_{|Q(\lambda)|>|\bar{B}|} \alpha_{\lambda}\left(\psi_{\lambda}(x)-\psi_{\lambda}\left(x_{0}\right)\right)$ converges absolutely in $X$, uniformly on $\bar{B}$; thus $\sum_{|Q(\lambda)|>|\bar{B}|} \alpha_{\lambda} \psi_{\lambda}(x)$ converges unconditionally on $L^{p}(\bar{B}, X) / X$.

The asserted convergence of $\sum \alpha_{\lambda} \psi_{\lambda}(x)$ has now been established. Moreover, the estimates (4.3) and (4.4) combined give

$$
\int_{\bar{B}}\left|\sum_{|Q(\lambda)| \leq|\bar{B}|} \alpha_{\lambda} \psi_{\lambda}(x)+\sum_{|Q(\lambda)|>|\bar{B}|} \alpha_{\lambda}\left(\psi_{\lambda}(x)-\psi_{\lambda}\left(x_{0}\right)\right)\right|_{X}^{p} d x \leq C \kappa^{p}|\bar{B}|,
$$

which shows the membership of the limit element in $\mathrm{BMO}\left(\mathbb{R}^{n}, X\right)$, and the asserted norm estimate.

Finally, we wish to exploit the wavelet framework to give a new point of view on the $H^{1}$-BMO duality in the UMD-valued situation. It should be noted that C. Fefferman's duality theorem [12] holds in the vector-valued situation under much milder geometric assumptions (see O. Blasco [1]), but requires a different approach.

4.5. Proposition. Let $X$ (and then also $X^{\prime}$ ) be a UMD space and $\left(\psi_{\lambda}\right)_{\lambda \in \Lambda}$ (and then also $\left.\left(\bar{\psi}_{\lambda}\right)_{\lambda \in \Lambda}\right)$ a 1-regular wavelet basis of $L^{2}\left(\mathbb{R}^{n}\right)$. Let

$$
b(x)=\sum_{\lambda \in \Lambda} \alpha_{\lambda}^{\prime} \bar{\psi}_{\lambda}(x) \in \operatorname{BMO}\left(\mathbb{R}^{n}, X^{\prime}\right), \quad \alpha_{\lambda}^{\prime}=\left\langle b, \psi_{\lambda}\right\rangle \in X^{\prime},
$$

where the convergence is unconditional in $L_{\mathrm{loc}}^{p}\left(\mathbb{R}^{n}, X^{\prime}\right) / X^{\prime}$. Then

$$
A(f)=A\left(\sum_{\lambda \in \Lambda} \alpha_{\lambda} \psi_{\lambda}\right):=\sum_{\lambda \in \Lambda} \alpha_{\lambda}^{\prime}\left(\alpha_{\lambda}\right)
$$

converges unconditionally for every $f=\sum_{\lambda \in \Lambda} \alpha_{\lambda} \psi_{\lambda} \in H^{1}\left(\mathbb{R}^{n}, X\right)$, and defines an element of $H^{1}\left(\mathbb{R}^{n}, X\right)^{\prime}$ with $\|A\|_{H^{1}\left(\mathbb{R}^{n}, X\right)^{\prime}} \leq C\|b\|_{\mathrm{BMO}\left(\mathbb{R}^{n}, X^{\prime}\right)}$.

Conversely, every $A \in H^{1}\left(\mathbb{R}^{n}, X\right)^{\prime}$ is of the form (4.6), where $\sum_{\lambda \in \Lambda} \alpha_{\lambda}^{\prime} \bar{\psi}_{\lambda}$ converges in $L_{\mathrm{loc}}^{p}\left(\mathbb{R}^{n}, X^{\prime}\right) / X^{\prime}$ to $b \in \mathrm{BMO}\left(\mathbb{R}^{n}, X\right)$ which satisfies $\|b\|_{\mathrm{BMO}\left(\mathbb{R}^{n}, X^{\prime}\right)}$ $\leq C\|A\|_{H^{1}\left(\mathbb{R}^{n}, X\right)^{\prime}}$. 
Proof. Let $F \subset \Lambda$ be finite. Then

$$
\sum_{\lambda \in F} \alpha_{\lambda}^{\prime}\left(\alpha_{\lambda}\right)=\int_{\mathbb{R}^{n}}\left\langle\sum_{\lambda \in F} \alpha_{\lambda}^{\prime} \bar{\psi}_{\lambda}(x), \sum_{\mu \in F} \alpha_{\mu} \psi_{\mu}(x)\right\rangle d x .
$$

According to Prop. 4.1, the $\operatorname{BMO}\left(\mathbb{R}^{n}, X\right)$ norms of $b_{F}:=\sum_{\lambda \in F} \alpha_{\lambda}^{\prime} \bar{\psi}_{\lambda}$ are bounded by $C\|b\|_{\mathrm{BMO}\left(\mathbb{R}^{n}, X\right)}$ for all $F \subset \Lambda$. On the other hand, from Theorem 1.9 it follows that the $H^{1}\left(\mathbb{R}^{n}, X\right)$ norms of $f_{F}:=\sum_{\mu \in F} \alpha_{\mu} \psi_{\mu}$ are uniformly bounded, and also that $\left\|f_{F}\right\|_{H^{1}\left(\mathbb{R}^{n}, X\right)}$ can be made smaller than any positive $\epsilon$ as soon as $F \subset F_{\epsilon}^{c}$, where $F_{\epsilon}$ is a sufficiently large set.

Now $f_{F}$ has an atomic decomposition $\sum a_{i}$, where $\operatorname{supp} a_{i} \subset \bar{B}_{i}, \int a_{i}=0$, and $\sum\left\|a_{i}\right\|_{L^{p^{\prime}\left(\mathbb{R}^{n}, X\right)}}\left|\bar{B}_{i}\right|^{1 / p} \leq 2\|f\|_{H^{1}\left(\mathbb{R}^{n}, X\right)}$. Since the atomic series converges in $L^{1}\left(\mathbb{R}^{n}, X\right)$, and $b_{F} \in L^{\infty}\left(\mathbb{R}^{n}, X^{\prime}\right)$, we have

$$
\begin{aligned}
\left|\left\langle b_{F}, f_{F}\right\rangle\right| & \leq \sum_{i=1}^{\infty}\left|\left\langle b_{F}, a_{i}\right\rangle\right| \leq \sum_{i=1}^{\infty}\left\|b_{F}\right\|_{\mathrm{BMO}\left(\mathbb{R}^{n}, X^{\prime}\right)}\left|\bar{B}_{i}\right|^{1 / p}\left\|a_{i}\right\|_{L^{p^{\prime}\left(\mathbb{R}^{n}, X\right)}} \\
& \leq 2 C\|b\|_{\mathrm{BMO}\left(\mathbb{R}^{n}, X^{\prime}\right)}\left\|f_{F}\right\|_{H^{1}\left(\mathbb{R}^{n}, X\right)},
\end{aligned}
$$

where a standard estimate for the pairing of a BMO function and an $H^{1}$ atom was used in the second step.

From this estimate and the unconditional convergence of $f_{F}$ to $f$ in $H^{1}\left(\mathbb{R}^{n}, X\right)$ as $F \uparrow \Lambda$, it follows that $\sum_{\lambda \in \Lambda} \alpha_{\lambda}^{\prime}\left(\alpha_{\lambda}\right)$ converges unconditionally to a complex number of absolute value at most $\|b\|_{\mathrm{BMO}\left(\mathbb{R}^{n}, X^{\prime}\right)}\|f\|_{H^{1}\left(\mathbb{R}^{n}, X\right)}$. This proves the first assertion.

The converse implication. Let now $A \in H^{1}\left(\mathbb{R}^{n}, X\right)^{\prime}$ be arbitrary. Define $\alpha_{\lambda}^{\prime} \in X^{\prime}$ by $\alpha_{\lambda}^{\prime}(x):=A\left(x \psi_{\lambda}\right)$ for $x \in X$. Since $\sum_{\lambda \in \Lambda} \alpha_{\lambda} \psi_{\lambda}$ converges unconditionally to $f$ in $H^{1}\left(\mathbb{R}^{n}, X\right)$, it follows that $\sum_{\lambda \in \Lambda} A\left(\alpha_{\lambda} \psi_{\lambda}\right)=\sum_{\lambda \in \Lambda} \alpha_{\lambda}^{\prime}\left(\alpha_{\lambda}\right)$ converges unconditionally to $A(f)$. Set $b_{F}:=\sum_{\lambda \in F} \alpha_{\lambda}^{\prime} \bar{\psi}_{\lambda}$ for finite $F \subset \Lambda$.

We estimate the $\operatorname{BMO}\left(\mathbb{R}^{n}, X\right)$ norm of $b_{F}$. Let $\bar{B}$ be a ball, and $f \in$ $L^{p^{\prime}}(\bar{B}, X)$. Then

$$
\left\langle b_{F}-\left(b_{F}\right)_{\bar{B}}, f\right\rangle=\left\langle b_{F}, f-f_{\bar{B}} 1_{\bar{B}}\right\rangle-\left\langle\left(b_{F}\right)_{\bar{B}} 1_{\bar{B}}, f\right\rangle+\left\langle b_{F}, f_{\bar{B}} 1_{\bar{B}}\right\rangle,
$$

and the last two terms are both equal to $|\bar{B}|\left\langle\left(b_{F}\right)_{\bar{B}}, f_{\bar{B}}\right\rangle$. Furthermore, note that $\left\langle b_{F}, g\right\rangle=\left\langle b_{F}, g_{F}\right\rangle=A\left(g_{F}\right)$ for any $g \in H^{1}\left(\mathbb{R}^{n}, X\right)$. Thus

$$
\begin{aligned}
\left|\left\langle b_{F}-\left(b_{F}\right)_{\bar{B}}, f\right\rangle\right| & =\left|A\left(\left(f-f_{\bar{B}} 1_{\bar{B}}\right)_{F}\right)\right| \leq\|A\|_{H^{1}\left(\mathbb{R}^{n}, X\right)^{\prime}}\left\|\left(f-f_{\bar{B}} 1_{\bar{B}}\right)_{F}\right\|_{H^{1}\left(\mathbb{R}^{n}, X\right)} \\
& \leq\|A\|_{H^{1}\left(\mathbb{R}^{n}, X\right)^{\prime}}\left\|f-f_{\bar{B}} 1_{\bar{B}}\right\|_{L^{p^{\prime}\left(\mathbb{R}^{n}, X\right)}}|\bar{B}|^{1 / p} .
\end{aligned}
$$

Taking the supremum over all $f \in L^{p^{\prime}}(\bar{B}, X)$ of norm at most 1 , and observing that the unit ball of $L^{p^{\prime}}(\bar{B}, X)$ is norming for $L^{p}\left(\bar{B}, X^{\prime}\right)$, we deduce

$$
\left\|\left(b_{F}-\left(b_{F}\right)_{\bar{B}}\right) 1_{\bar{B}}\right\|_{L^{p}\left(\mathbb{R}^{n}, X^{\prime}\right)} \leq 2\|A\|_{H^{1}\left(\mathbb{R}^{n}, X\right)^{\prime}}|\bar{B}|^{1 / p},
$$

and thus $\left\|b_{F}\right\|_{\mathrm{BMO}\left(\mathbb{R}^{n}, X\right)} \leq 2\|A\|_{H^{1}\left(\mathbb{R}^{n}, X\right)^{\prime}}$. From Prop. 4.1 it follows that this uniform estimate for $b_{F}$ implies that $b_{F} \rightarrow b$ as $F \uparrow \Lambda$, unconditionally 
in the space $L_{\text {loc }}^{p}\left(\mathbb{R}^{n}, X^{\prime}\right) / X^{\prime}$, and $\|b\|_{\mathrm{BMO}\left(\mathbb{R}^{n}, X^{\prime}\right)} \leq C\|A\|_{H^{1}\left(\mathbb{R}^{n}, X\right)}$. Then, by the first part of the proof, $b$ defines via duality an element $\widetilde{A} \in H^{1}\left(\mathbb{R}^{n}, X\right)^{\prime}$. It is clear that $\widetilde{A}(f)=\left\langle b_{F}, f\right\rangle=A(f)$ if $f=\sum_{\lambda \in F} \alpha_{\lambda} \psi_{\lambda}$ and $F \subset \Lambda$ is finite; since such $f$ are dense in $H^{1}\left(\mathbb{R}^{n}, X\right)$, we see that $A=\widetilde{A}$, i.e., $A$ is of the asserted form.

The previous proposition shows that $H^{1}\left(\mathbb{R}^{n}, X\right)^{\prime}=\mathrm{BMO}\left(\mathbb{R}^{n}, X^{\prime}\right)$ for $X$ UMD, which, as already mentioned, actually holds under more general conditions. While restricted to the UMD setting, the present approach has the virtue of providing the explicit formula (4.6) for the evaluation of the duality pairing $\langle b, f\rangle$. Note that the wavelet coefficients $\alpha_{\lambda}^{\prime}$ of $b$ and $\alpha_{\lambda}$ of $f$ are uniquely determined by the functions $b$ and $f$, and moreover explicitly given by the formulae $\alpha_{\lambda}^{\prime}=\left\langle b, \psi_{\lambda}\right\rangle, \alpha_{\lambda}=\left\langle f, \bar{\psi}_{\lambda}\right\rangle$. On the other hand, the atomic decomposition of $f$, in terms of which the $H^{1}$-BMO duality is often defined by $\langle b, f\rangle=\sum_{i=1}^{\infty}\left\langle b, a_{i}\right\rangle$ is far from being unique.

From the previous proof we also readily see the following, recalling that UMD spaces are reflexive:

4.8. Corollary. Let $X$ be a UMD space, and $\left(\psi_{\lambda}\right)_{\lambda \in \Lambda}$ a 1-regular wavelet basis. Then, for every $b \in \mathrm{BMO}\left(\mathbb{R}^{n}, X\right)$, the wavelet expansions $\sum_{\lambda \in F}\left\langle b, \bar{\psi}_{\lambda}\right\rangle \psi_{\lambda}$ converge unconditionally to $b$ in the weak $k^{*}$ topology $\sigma\left(\mathrm{BMO}\left(\mathbb{R}^{n}, X\right), H^{1}\left(\mathbb{R}^{n}, X^{\prime}\right)\right)$ as $F \uparrow \Lambda$.

\section{References}

[1] O. Blasco, Hardy spaces of vector-valued functions: duality, Trans. Amer. Math. Soc. 308 (1988), 495-507.

[2] J. Bourgain, Some remarks on Banach spaces in which martingale difference sequences are unconditional, Ark. Mat. 21 (1983), 163-168.

[3] -, Vector-valued singular integrals and the $H^{1}-\mathrm{BMO}$ duality, in: J. A. Chao and W. Woyczyński (eds.), Probability Theory and Harmonic Analysis, Dekker, New York, 1986, 1-19.

[4] D. L. Burkholder, A geometric condition that implies the existence of certain singular integrals of Banach-space-valued functions, in: Conf. in honor of A. Zygmund (Chicago, 1981), Wadsworth Math. Ser., Wadsworth, 1983, 270-286.

[5] D. L. Burkholder, Martingales and Fourier analysis in Banach spaces, in: G. Letta and M. Pratelli (eds.), Probability and Analysis, Lecture Notes in Math. 1206, Springer, 1986, 61-108.

[6] A. P. Calderón, An atomic decomposition of distributions in parabolic $H^{p}$ spaces, Adv. Math. 25 (1977), 216-225.

[7] S.-Y. A. Chang and Z. Ciesielski, Spline characterizations of $H^{1}$, Studia Math. 75 (1983), 183-192.

[8] S.-Y. A. Chang and R. Fefferman, A continuous version of duality of $H^{1}$ with BMO on the bidisc, Ann. of Math. (2) 112 (1980), 179-201.

[9] - - - The Calderón-Zygmund decomposition on product domains, Amer. J. Math. 104 (1982), 455-468. 
[10] G. David and J.-L. Journé, A boundedness criterion for generalized CalderónZygmund operators, Ann. of Math. 120 (1984), 371-397.

[11] B. Davis, On the integrability of the martingale square function, Israel J. Math. 8 (1970), 187-190.

[12] C. Fefferman, Characterizations of bounded mean oscillation, Bull. Amer. Math. Soc. 77 (1971), 587-588.

[13] T. Figiel, On equivalence of some bases to the Haar system in spaces of vector-valued functions, Bull. Pol. Acad. Sci. Math. 36 (1988), 119-131.

[14] T. Figiel, Singular integral operators: a martingale approach, in: P. F. X. Müller and W. Schachermayer (eds.), Geometry of Banach Spaces (Strobl, 1989), London Math. Soc. Lecture Note Ser. 158, Cambridge Univ. Press, 1990, 95-110.

[15] T. Figiel and P. Wojtaszczyk, Special bases in function spaces, in: W. B. Johnson and J. Lindenstrauss (eds.), Handbook of the Geometry of Banach Spaces, Vol. I, Elsevier, 2001, 561-597.

[16] J.-L. Journé, Calderón-Zygmund Operators, Pseudo-Differential Operators and the Cauchy Integral of Calderón, Lecture Notes in Math. 994, Springer, 1983.

[17] B. Maurey, Système de Haar, in: Séminaire Maurey-Schwartz 1974-1975: Espaces $L^{p}$, applications radonifiantes et géométrie des espaces de Banach, Exp. Nos. I et II, Centre Math., École Polytech., Paris, 1975.

[18] Y. Meyer, Wavelets and Operators, Cambridge Univ. Press, 1992.

[19] Y. Meyer and R. Coifman, Wavelets: Calderón-Zygmund and Multilinear Operators, Cambridge Univ. Press, 1997.

[20] P. F. X. Müller and G. Schechtman, Several results concerning unconditionality in vector valued $L^{p}$ and $H^{1}\left(\mathcal{F}_{n}\right)$ spaces, Illinois J. Math. 35 (1991), 220-233.

[21] E. M. Stein, Harmonic Analysis: Real-Variable Methods, Orthogonality, and Oscillatory Integrals, Princeton Math. Ser. 43, Princeton Univ. Press, 1993.

Department of Mathematics

University of Turku

FI-20014 Turku, Finland

E-mail: tuomas.hytonen@utu.fi

Received October 6, 2003

Revised version October 26, 2005 